\title{
The phosphatidylinositol 3-kinase/Akt and c-Jun N-terminal kinase signaling in cancer: Alliance or contradiction? (Review)
}

\author{
HUA-FU ZHAO ${ }^{1}$, JING WANG ${ }^{2}$ and SHING-SHUN TONY TO ${ }^{1}$ \\ ${ }^{1}$ Department of Health Technology and Informatics, The Hong Kong Polytechnic University, Hong Kong; \\ ${ }^{2}$ Department of Neurosurgery/Neuro-oncology, Sun Yat-sen University Cancer Center; State Key Laboratory of Oncology \\ in South China; Collaborative Innovation Center for Cancer Medicine, Guangzhou 510060, P.R. China
}

Received March 7, 2015; Accepted April 27, 2015

DOI: 10.3892/ijo.2015.3052

\begin{abstract}
The phosphatidylinositol 3-kinase (PI3K)/Akt signaling pathway and c-Jun N-terminal kinase (JNK) pathway are responsible for regulating a variety of cellular processes including cell growth, migration, invasion and apoptosis. These two pathways are essential to the development and progression of tumors. The dual roles of JNK signaling in apoptosis and tumor development determine the different interactions between the PI3K/Akt and JNK pathways. Activation of PI3K/ Akt signaling can inhibit stress- and cytokine-induced JNK activation through Akt antagonizing and the formation of the JIP1-JNK module, as well as the activities of upstream kinases ASK1, MKK4/7 and MLK. On the other hand, hyperactivation of Akt and JNK is also found in cancers that harbor EGFR overexpression or loss of PTEN. Understanding the activation mechanism of PI3K/Akt and JNK pathways, as well as the interplays between these two pathways in cancer may contribute to the identification of novel therapeutic targets. In the present report, we summarized the current understanding of the PI3K/Akt and JNK signaling networks, as well as their biological roles in cancers. In addition, the interactions and regulatory network between PI3K/Akt and JNK pathways in cancer were discussed.
\end{abstract}

\section{Contents}

1. Introduction

2. Canonical PI3K/Akt signaling pathway

3. Network of c-Jun $\mathrm{N}$-terminal kinase (JNK) signaling pathway

4. Dual roles of JNK signaling in apoptosis and tumor development

Correspondence to: Dr Shing-Shun Tony To, Department of Health Technology and Informatics, The Hong Kong Polytechnic University, Hong Kong, P.R. China

E-mail: tony.to@polyu.edu.hk

Key words: PI3K/Akt, JNK, PTEN, EGFR, crosstalk, apoptosis, tumor development
5. Crosstalk between PI3K/Akt and JNK pathways

6. Conclusion

\section{Introduction}

The phosphatidylinositol 3-kinase (PI3K)/Akt signaling pathway plays a pivot role in the development and survival of cancers. The upstream regulators and downstream effectors of this pathway consists of a complex cellular signaling network including crosstalk, feedback loops, and branch points that controls a variety of cellular processes and functions. One of the essential downstream signaling pathways of Akt is the c-Jun N-terminal kinase (JNK) signaling pathway, which belongs to a subgroup of mitogen-activated protein kinase (MAPK) signaling pathways. The interaction between PI3K/Akt and JNK pathways is complicated due to the dual roles of JNK signaling in apoptosis. The interaction between these two pathways may determine the fate of the cell: survival or apoptosis.

In this review, we first summarized the functional characteristics, signal transduction and activation mechanisms of the PI3K/Akt and JNK pathways, and then discussed the dual roles of JNK pathway in apoptosis and tumor development. Upon this background, the interaction between these two signaling pathways was mapped to determine the potential targets and combination therapeutic strategies for cancer treatment.

\section{Canonical PI3K/Akt signaling pathway}

PI3Ks are lipid kinases involved in a variety of biological processes, such as cell proliferation, differentiation, motility, survival and angiogenesis $(1,2)$. PI3Ks are activated by receptor tyrosine kinases (RTKs) or G-protein-coupled receptors (GPCRs). RTKs include a variety of cell surface receptors that interact with growth factors, cytokines, or hormones including insulin, epidermal growth factor (EGF), plateletderived growth factor (PDGF), and insulin-like growth factor-1 (IGF-1). Under the circumstance of ligand stimulation, RTKs undergo autophosphorylation, providing binding sites for PI3K regulatory subunits which subsequently lead to PI3K activation. Activated PI3K phosphorylates the lipid 


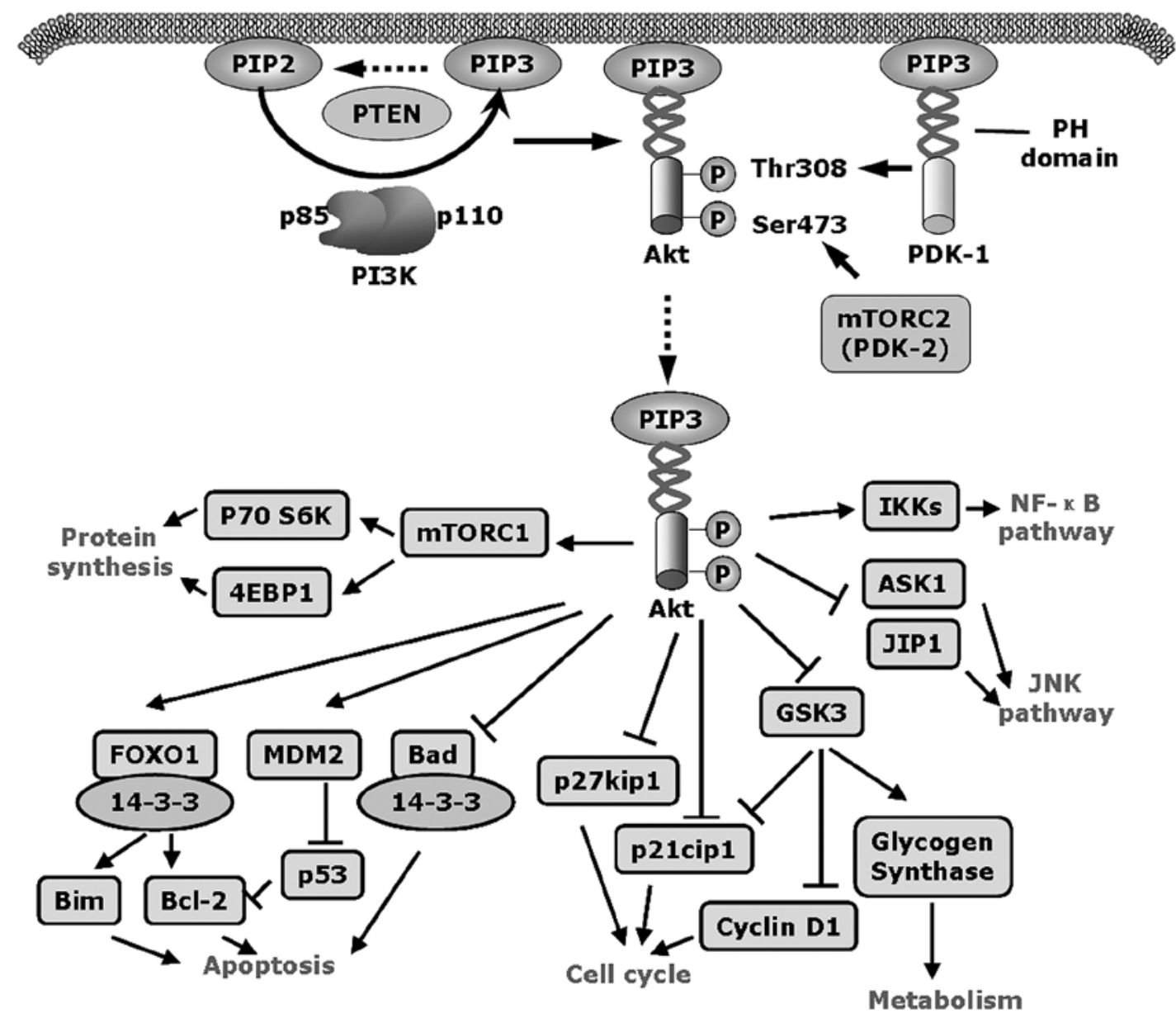

Figure 1. Simplified scheme showing the process of Akt activation and its downstream effectors. Activated PI3Ks phosphorylate PIP2 to PIP3, leading to binding of PIP3 to the PH domain of Akt and recruitment of Akt to the plasma membrane. Tumor suppressor PTEN can dephosphorylate PIP3 back to PIP2 and inactivate Akt. PIP3 also recruits phosphoinositide-dependent kinase-1 (PDK-1) to the plasma membrane through binding to the PH domain of PDK-1. PDK-1 then phosphorylates Akt at Thr308 in the activation loop to partially activate Akt. Subsequently, Akt is completely activated through phosphorylation at Ser473 by another activating kinase termed PDK-2. It is believed that PDK-2 is mTORC2 consisting of mTOR and Rictor. Activated Akt and PDK-1 can phosphorylate a variety of downstream effectors including mTOR, GSK3, Bad, IKK and FOXO1 to regulate cellular growth, proliferation, motility, survival and metabolism.

phosphatidylinositol 4,5-bisphosphate [PtdIns(4,5) $\left.\mathrm{P}_{2}, \mathrm{PIP} 2\right]$ to generate phosphatidylinositol 3,4,5-triphosphate $[\operatorname{Ptd} \operatorname{Ins}(3,4,5)$ $\left.\mathrm{P}_{3}, \mathrm{PIP} 3\right]$, which then binds to the Pleckstrin homology (PH) domain of Akt and recruits it to the plasma membrane (Fig. 1). PIP3 also engages phosphoinositide-dependent kinase-1 (PDK-1) to plasma membrane through binding to its $\mathrm{PH}$ domain. PDK-1 then phosphorylates Akt at residue threonine 308 (Thr308) in the activation loop to partially activate Akt $(3,4)$. Subsequently Akt is completely activated through phosphorylation at serine 473 (Ser473) in the hydrophobic motif by mTOR complex 2 (mTORC2) (5). Activated Akt and PDK-1 can phosphorylate a number of downstream proteins such as mTOR, glycogen synthase kinase 3 (GSK3), Bcl-2associated death promoter (Bad) and forkhead box protein O1 (FOXO1) to regulate cell growth, motility, survival and metabolism (Fig. 1) (6).

Phosphatase and tensin homolog deleted on chromosome 10 (PTEN) is a tumor suppressor with lipid phosphatase and tyrosine phosphatase activities. Wild-type PTEN is able to dephosphorylate PIP3 back to PIP2, and interfere with the recruitment of Akt to the plasma membrane, resulting in reduced Akt activation $(7,8)$. PTEN locates on chromosome $10 \mathrm{q} 23$, which is highly susceptible to mutation in human cancers. Mutation or loss of function of PTEN is frequent in a wide range of cancers including glioblastoma multiforme (9), gastric cancer (10), endometrial cancer $(11,12)$, ovarian cancer (12) and lung cancer (13). PTEN can decrease the synthesis of IGF-I, -II and IGF-1R, which in turn generates an autocrine loop to downregulate Akt activation $(14,15)$. Therefore, high Akt phosphorylation is frequently associated with loss of PTEN, leading to chemorestistance and poor prognosis in cancer patients $(16,17)$. Convincing evidence shows that PTEN inactivation confers higher EGFR phosphorylation in tumor cells and their resistance to EGFR specific inhibitors (18). Wild-type PTEN promotes the ubiquitination of activated EGFR through PIP3 and Akt-dependent mechanism (18). These findings indicate that PTEN is a key element of PI3K/Akt pathway and acts as an important tumor suppressor through suppressing PI3K/Akt signaling.

\section{Network of c-Jun N-terminal kinase (JNK) signaling pathway}

JNK, also known as stress-activated protein kinase (SAPK), can be activated in response to a number of environmental challenges including UV irradiation, heat shock, toxins, as 


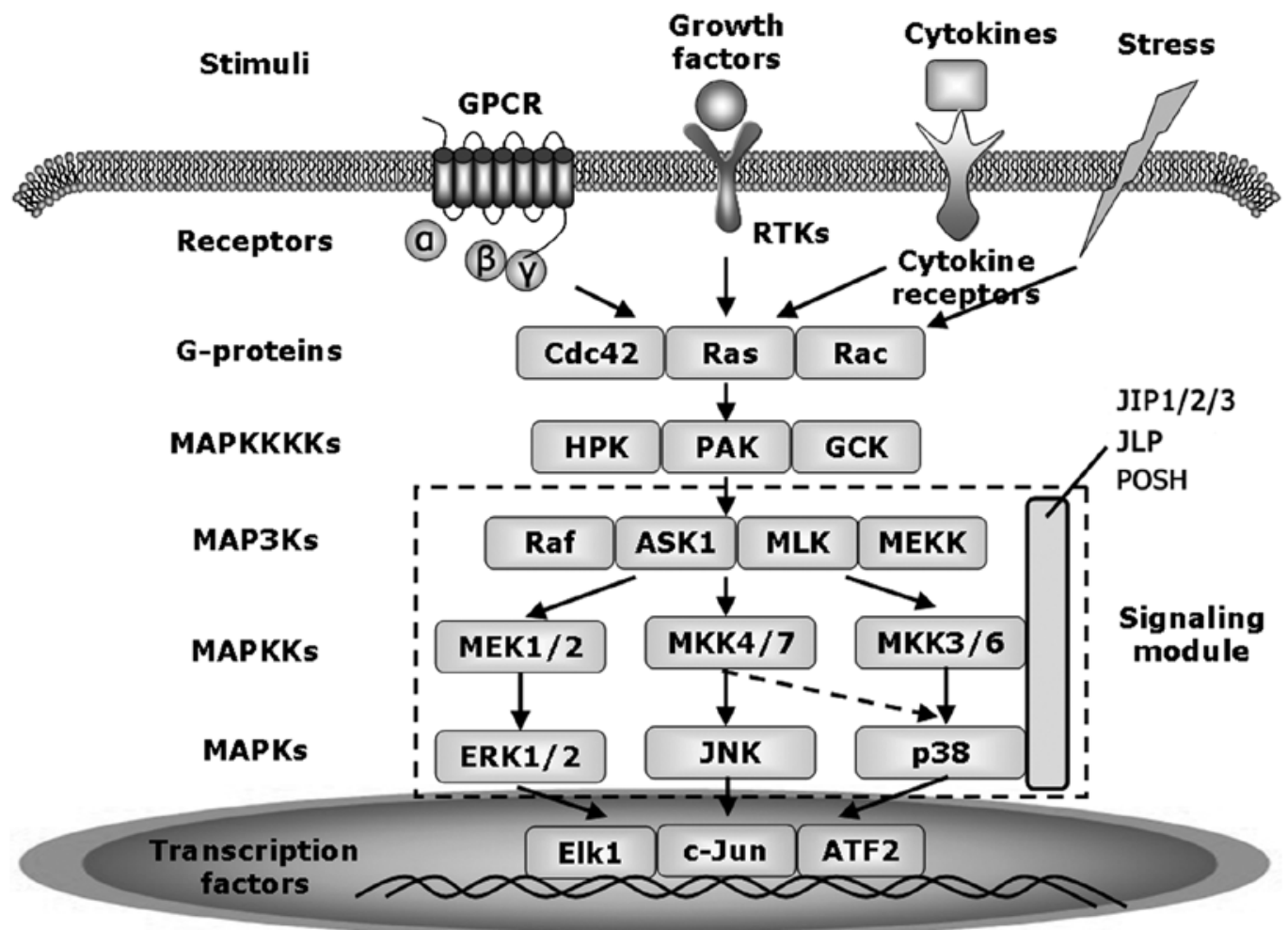

Figure 2. Simplified pathway of mitogen-activated protein kinases (MAPKs). The main MAPK families include ERK, JNK and p38, which are activated by stress, cytokines and growth factors. Generally, activation of MAPK signaling pathways starts with receptor activation, leading to the activation of small GTPbinding proteins (G-proteins) such as Cdc42, Ras and Rac. The protein kinase cascade that consists of up to four tiers of kinases is subsequently activated. The scaffold proteins including JIP, JLP and POSH interact with specific members of MAP3Ks, MAPKKs and MAPKs to form a module that facilitates signaling. The activated MAPKs translocate to the nucleus and phosphorylate specific transcription factors including ETS-like transcription factor 1 (ELK1), c-Jun and activating transcription factor-2 (ATF2).

well as cytokines and growth factors (19). In mammals, JNK is encoded by three genes (JNK1, JNK2 and JNK3), which are located on three different chromosomes. Among these three genes, JNK1 and $J N K 2$ are ubiquitously expressed, while $J N K 3$ is largely restricted to the brain, heart and testis (20). This family has at least ten isoforms: $J N K 1$ (four isoforms), $J N K 2$ (four isoforms) and $J N K 3$ (two isoforms), which are generated by the alternative splicing of the mRNA 3'-coding region. These $\mathrm{JNK}$ isoforms are expressed as $54 \mathrm{kDa}$ and $46 \mathrm{kDa}$ proteins that recognize and interact with different substrates. Although the function of JNK splice variants is not clear so far, it is found that JNK-interacting protein 1 (JIP1) can augment the expression of $46 \mathrm{kDa}$ of JNK splice variants and increase the stability of JNK-JIP1 module (21). It indicates that JNK variants have different affinity to the scaffold proteins, which may result in the JNK variants recognizing different substrates. JNK is a member of an evolutionarily conserved sub-family of mitogen-activated protein kinases (MAPKs). In mammals, several members of MAPK family have been identified, including extracellular signal-regulated kinases (ERKs), SAPK/JNK, the 38-kDa protein kinases (p38), and ERK5/ big mitogen activated protein kinase 1 (BMK1). Generally, activation of MAPK signaling pathways starts with receptor activation, leading to the recruitment of adaptor proteins and activation of small GTP-binding proteins (G-proteins) (22). The protein kinase cascade that consists of up to four tiers of kinases is subsequently activated. The MAPKKKKs phos- phorylate and activate the MAPKKKs (MAP3Ks or MEKKs) which, in turn, phosphorylate and activate the MAPKKs (or MEKs). MAPKKs then phosphorylate and activate the MAPKs, which phosphorylate and interact with their specific substrates (Fig. 2) (23).

JNK activation is mediated by two upstream MAPKKs: mitogen-activated protein kinase kinase 4 (MKK4) (also known as SAPK/ERK kinase 1, SEK1) and MKK7. MKK4 and MKK7 preferentially phosphorylate JNK at tyrosine and threonine residues respectively, leading to full activation of JNK (Fig. 2) (24,25). MAP3Ks that activate MKK4 and MKK7 in JNK signaling pathway, include the Raf kinase (26), apoptosis signal-regulating kinase-1 (ASK1) (27), MAPK kinase kinase 1/4 (MEKK1/4) (28) and mixed-lineage kinase (MLK) (29). MAP3Ks are activated by Rho family of GTPases, a family of small signaling $\mathrm{G}$ proteins ( $21 \mathrm{kDa})$ including the cell division control protein 42 (CDC42), Rac, Ras and Ras homolog gene family member A (RhoA) (30). Activated JNK can translocate into the nucleus and phosphorylate a variety of transcription factors including c-Jun, JunB, JunD, ATF2, STAT3 and p53 (Fig. 2). The first identified substrate of JNK is c-Jun, of which the activation and stability are mediated by phosphorylation of Ser63 and Ser73 in the N-terminal region. Further, phosphorylated c-Jun can interact with c-Fos to form activator protein-1 (AP-1) complex, which can specifically bind to the promoter or enhancer of numerous genes to mediate their transcriptional activity (31). 
Several proteins such as JNK interacting protein (JIP), JNK-interacting leucine zipper protein (JLP), and plenty of SH3 (POSH) have been identified as scaffold proteins that interact with specific member of JNK signaling cascade and form a module to facilitate signaling transduction (Fig. 2). The first identified scaffold protein in JNK signaling pathway is JIP1, which only interacts directly with SAPK/JNK, rather than other MAPKs, p38 and ERK (32). JIP1 recruits JNK, MKK7, MLK, and haematopoietic progenitor kinase-1 (HPK1) to form the signaling module (33). The activation of the JNK module requires the phosphorylation of JIP1 by JNK (34). However, overexpression of JIP-1 could retain JNK in the cytoplasm and prevent the activation of c-Jun and activating transcription factor 2 (ATF2), indicating that excess JIP1 may decrease JNK activity through a negative feedback loop (32).

\section{Dual roles of JNK signaling in apoptosis and tumor development}

The JNK signaling pathway controls a variety of cellular events including cell proliferation, development, inflammation, and apoptosis. JNK can act as a pro-apoptotic or anti-apoptotic molecule depending on the circumstance. Evidence shows that apoptosis is mediated by JNK signaling in a stimulus-dependent manner $(35,36)$. Numerous studies have demonstrated that the MLK/JNK/c-Jun axis promotes apoptosis not only in sympathetic neurones $(37,38)$, but also in cancer cells (39-41). Knockdown of dual leucine zipper-bearing kinase (DLK), a member of the MLK family, is able to suppress JNK phosphorylation and poly-ADP ribose polymerase (PARP) cleavage, leading to inhibition of calphostin C-induced apoptosis in human breast cancer cells (40). The JNK inhibitor SP600125 inhibits the phosphorylation of BCL2-associated X protein (Bax) and prevents the translocation of JNK into mitochondria, resulting in suppression of stress-induced apoptosis in human hepatoma cells (42). In addition, JNK is able to promote apoptosis by regulating p53 upregulated modulator of apoptosis (PUMA) $(43,44)$. PUMA is a pro-apoptotic BH3-only protein that promotes stress- and growth factor-induced apoptosis in either p53-dependent or -independent manner, through direct interaction with B-cell lymphoma 2 (Bcl-2) family members and antagonizing the anti-apoptotic effect of Bcl-2 family (45). JNK effectively enhances PUMA activation and apoptosis induced by betulinic acid in cisplatin-resistant ovarian cancer cells (44). Recent studies also found that p53 and its homologue p73 are the substrates of JNK and can be phosphorylated by JNK $(46,47)$. Phosphorylated p53 or p73 subsequently binds to the promoter of PUMA and regulates its expression. These findings indicate that JNK may be a critical upstream regulator of PUMA and plays a pro-apoptotic role in cancer cells in the context of a wide variety of stimuli including genotoxic stress, cytokines and growth factors.

Activation of JNK is also involved in cell survival and antiapoptosis in both Fas- and mitochondria-dependent manner $(48,49)$. Evidence shows that thymocytes and peripheral $\mathrm{T}$ cells from $M K K 4^{-/}$mice are more susceptible to CD95 (Fas)and CD3-mediated apoptosis, indicating MKK4-induced JNK activation participates in the survival of T-cell (49). Expression of a constitutively active JNK mutant suppresses pro-B cell apoptosis induced by IL-3 withdrawal. JNK phosphorylates
BAD at Thr201, and prohibits BAD from interacting with Bcl-xL, resulting in decreased apoptosis (48). In addition, downregulation of JNK2 expression induces significant apoptosis in a variety of p53-deficient cancer cell lines (50).

The JNK pathway also plays different roles in tumor development. A number of studies report that JNK is required for tumor formation and development. Evidence shows that both JNK1 and JNK2 are required for in vitro Ras-induced cellular transformation and in vivo tumor formation of lung cancer, which is correlated with increased c-Jun and AP-1 activities (51-53). JNK2 can phosphorylate ATF-2 and further protect c-Myc from proteasomal degradation during Ras-induced transformation in mouse embryonic fibroblasts (MEFs) (54). Furthermore, c-Jun is also required for Ras-induced transformation. The $\mathrm{c}$-Jun ${ }^{-/}$fibroblasts do not show transformation induced by Ras, which can be reversed by overexpression of wild-type c-Jun (55). These findings indicate that JNK/c-Jun axis is essential in the Ras-induced transformation. The evidence that JNK pathway promotes cellular transformation supports that JNK signaling is also essential in tumor development. Recently, constitutively active isoforms of JNKs have been found in various cancers including gastric cancer (56), hepatocellular carcinoma (57), breast cancer (58) and glioma (59). Using an ATP-competitive JNK inhibitor SP600125, the growth of pancreatic cancer in vitro and in vivo were suppressed, and the survival of mice was also prolonged (60). In addition, decreased DNA damage and replicative stress response are found in $J N K 2^{-/}$mammary tumor mice, and $J N K 2^{-/-}$mice exhibit shorter latency and higher tumor multiplicity than wild-type $J N K 2$, indicating that JNK2 is required for tumor development and genetic stability (61).

Supporting evidence shows that deficiency of both JNK1 and JNK2 in hepatocytes promotes diethylnitrosamine (DEN)-induced hepatocellular carcinoma (HCC) development in mice, which may result from the anti-apoptotic role of JNK. Deficiency of JNK causes increased apoptosis of hepatocytes, leading to increased compensatory proliferation that contributes to HCC development. On the contrary, deficiency of JNK in nonparenchymal cells suppresses HCC development by increasing the expression of cytokines IL-6 and TNF- $\alpha$ to provide an inflammatory environment (62). It indicates that the role of JNK in tumor development is cell type-dependent.

Since the JNK pathway can also act a pro-apoptotic role in cancer, JNK may be considered as a tumor suppressor. Loss of JNK expression increases the number and growth of tumor nodules in vivo and induces the transformed phenotype of fibroblasts in vitro. Besides, JNK-null cells display less Ras-induced apoptosis than cells with wild-type JNK (63). Other studies also show that inactivation of JNK in the prostate epithelium results in rapid development of invasive adenocarcinoma in vivo (64). The JNK3 is considered as the tumor suppressor gene. Loss of $J N K 3$ gene is found in a variety of cancer cell lines including brain tumor (10 of 19), nonHodgkin's lymphoma (15 of 16), Hodgkin's lymphoma (3 of 6), breast cancer (3 of 10), gastric cancer (6 of 10), and hepatocellular carcinoma (8 of 12) $(65,66)$. Moreover, activation of JNK3, rather than JNK1 and JNK2, induces mitochondrial dysfunction and promotes TNF- $\alpha$-induced apoptosis in human oligodendrocytes, suggesting a possible mechanism for the tumor suppressor role of JNK3 (67). 

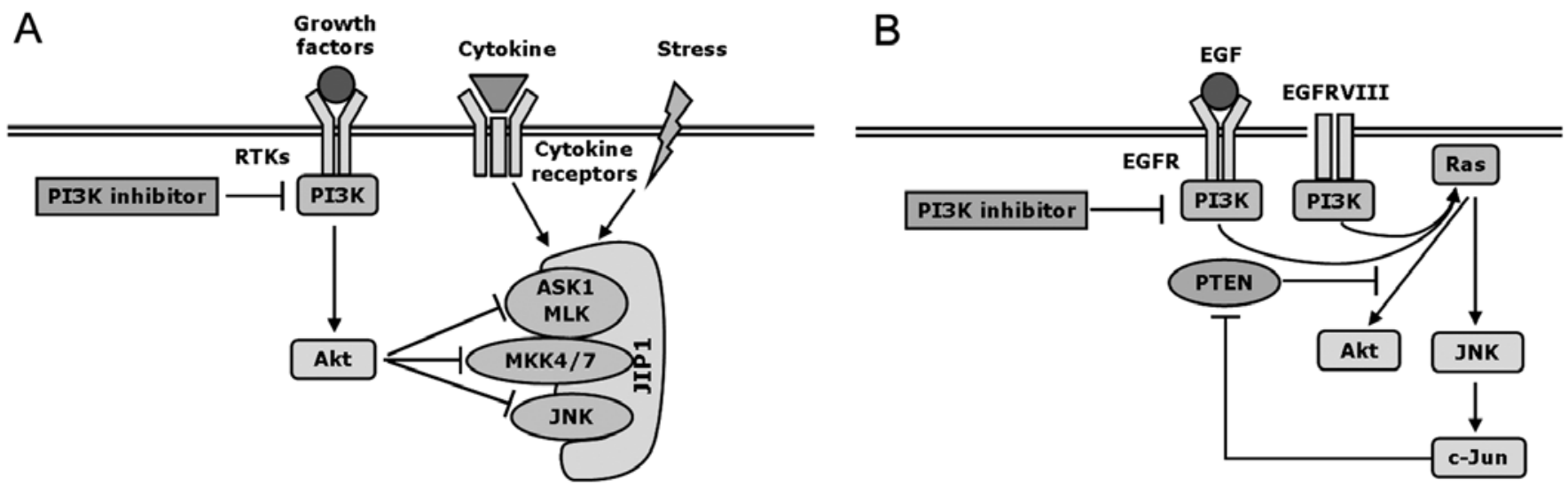

Figure 3. Simplified scheme demonstrating different interactions between PI3K/Akt and JNK pathways. (A) Activation of PI3K/Akt signaling inhibits stressand cytokine-induced JNK activation. JIP1 can directly bind to the PH domain of Akt1, leading to the formation of Akt1-JIP1 module and disassociation of JNK from JIP-JNK module. In addition, Akt interacts with ASK1, MLK3 and MKK4, and phosphorylates them at Ser83, Ser624 and Ser78 respectively to inhibit their kinase activities, leading to the inactivation of JNK. The PI3K inhibitor activates JNK signaling through inactivation of Akt. (B) The pattern of Akt and JNK co-activation is exhibited in the context of EGF stimulation, EGFR or EGFRVIII overexpression, as well as loss of PTEN. The PI3K inhibitors and wild-type PTEN expression are able to inhibit both Akt and JNK activation. Activated c-Jun by JNK binds to the promoter sequence of PTEN, resulting in the inhibition of PTEN transcription and activation of Akt.

\section{Crosstalk between PI3K/Akt and JNK pathways}

PI3K/Akt antagonizes stress- and cytokine-induced JNK signaling activation. Since the PI3K/Akt pathway plays essential roles in tumor transformation, development and progression, the dual roles of JNK signaling in apoptosis and tumor development may determine different crosstalk between PI3K/Akt and JNK pathways. Increasing evidence reveals that these two signaling pathways interact with each other and consist of a regulatory network. The scaffold protein JIP1, which is essential for formation and activation of JNK module, can directly bind to the $\mathrm{PH}$ domain of Akt1, leading to the formation of Akt1-JIP1 complex facilitating the activation of Akt1 via PDK1 (68-70). Moreover, increasing interaction between JIP1 and Akt1 leads to release of JNK from JIP-JNK module and inhibition of JNK activity $(70,71)$. Overexpression of wild-type or constitutively active Akt1 also inhibits JNK activity, especially JNK2, and decreases apoptosis in a variety of cells such as normal neurons, 293T, PC12 and T cells (70-73). On the contrary, since the activation of Akt requires the activities of PI3K or RTKs, withdrawal of RTK ligands such as insulin and IGF-1, or PI3K inhibitor treatment promotes JNK activation by dephosphorylating Akt and increasing the interaction between JIP1 and JNK (69,71-74).

Apart from interacting with JIP1, Akt can bind to ASK1 and phosphorylate ASK1 at Ser83 $(75,76)$. ASK1 is a member of MAP3Ks and serves as an upstream activator of JNK. Phosphorylation of Akt inhibits the oxidative stress-induced activation of ASK1 in human embryonic kidney 293T cells, leading to reduced apoptosis (76). In addition, IGF-1 stimulation suppresses the activation of ASK $1 / \mathrm{JNK}$ induced by serum deprivation or cytokines through activation of PI3K/Akt signaling, whereas PI3K inhibitors reverse the inhibitory effect (76,77). Recent evidence indicates that disabled-2 interacting protein (DAB2IP) is a scaffold protein bridging both Akt and ASK1 via different domains, and its overexpression suppresses Akt signaling but activates ASK1/JNK, leading to enhanced TNF- $\alpha$-induced apoptosis in prostate cancer cells (78). Besides,
MKK4 is also a substrate of Akt in intact cells. Activated Akt induced by insulin is able to phosphorylate MKK4 at Ser78 and inhibit the activation of MKK4 and JNK in 293T cells, leading to prolonged cell survival (79). In addition, angiopoietin-1-induced Akt activation also phosphorylates MKK4 at Ser80 and suppress apoptosis and oxidative stress-induced JNK activation in vascular endothelial cells (80). Furthermore, Akt interacts with and phosphorylate MLK3 on Ser674 to inhibit its kinase activity $(81,82)$. Insulin-induced phosphorylation of Akt concomitant with reduced kinase activities of MLK3, MKK7 and JNK is observed in human hepatoma HepG2 cells, indicating that activation of Akt antagonizes MLK3-MKK7-JNK signaling (81). Thus, JNK signaling plays a pro-apoptotic role, which is antagonized by Akt activation under stimuli including stress, toxin and cytokines (Fig. 3A).

Interestingly, Song and Lee found that PI3K/Akt signaling inhibits glucose deprivation induced-activation of JNK through antagonizing JIP1-MKK4-JNK cascade in human prostate carcinoma cells (83). However, activated JNK2 phosphorylates JIP1 at Thr103, leading to increased interaction between JIP1 and JNK2, and disassociation of Akt1 from JIP1. Subsequently, the disassociated Akt1 binds to MKK4 and inhibits its activity. The inhibition of MKK4 in turn suppresses the activation of JNK, leading to the formation of a negative regulatory feedback loop (83).

Co-activation of Akt and JNK signaling in cancer is correlated with EGFR overexpression and PTEN loss. JNK preferentially takes on a pro-apoptosis role in response to a variety of stimuli including stress, toxin and cytokines. Activation of PI3K/Akt signaling inhibits the JNK activity and leads to decreased apoptosis, which is consistent with the finding that PI3K/ Akt signaling is positively correlated with survival. However, a number of studies show that activation of Akt or PI3K is frequently accompanied by JNK activation in cancers including glioblastoma, cervical carcinoma and prostate cancer, indicating that JNK may play an anti-apoptotic role under certain circumstances (84-86). One possible explanation is that JNK 
is constitutively active in some types of cancers. Reports show that JNK2 isoforms are constitutively active in glioblastoma and non-small cell lung carcinoma, and a positive correlation between elevated JNK activity and higher histological grade is found (86-89). If JNK plays a pro-apoptotic role in apoptosis induced by extracellular stimuli, the constitutively activated JNK may served as an anti-apoptotic protein contributing to the survival of cancer cells. Another possible explanation is that activation of both Akt and JNK signaling can be induced by EGF/EGFR in cancers, which is correlated with the loss of PTEN. Overexpression of wild-type EGFR or EGFRVIII (a mutated variant of EGFR with constitutive activity), as well as EGF stimulation activate both PI3K/Akt and JNK signaling pathways in various cancer cell lines (90-92). Aberrant expression of EGFR or EGFRVIII in $P_{T E N^{-/}}$glioblastoma cells induces activation of JNK and transcription factor JunD/ AP-1, as well as activation of Akt. Restoration of PTEN, which antagonizes PI3K activity, diminishes activities of Akt and JNK (91). Using the PI3K inhibitor wortmannin or a dominantnegative mutant of PI3K, Akt phosphorylation is inhibited in HeLa cells, and the activation of JNK induced by EGF is also suppressed, whereas no effect is observed on the JNK activity induced by UV or osmotic stress (84). These findings suggest that EGF/EGFR and constitutively active EGFRVIII are the upstream activators of Akt and JNK signaling in cancers (Fig. 3B).

In addition, JNK activation is PI3K-dependent and promoted by loss of PTEN (Fig. 3B). Evidence reveals that activities of JNK and c-Jun are constitutively elevated in

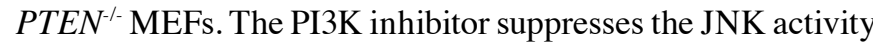
in $P T E N^{-/}$MEFs and prostate cancer cells, whereas the inhibitory effect is much less in MEFs and prostate cancer cells with wild-type PTEN (85). It is also found that PTEN inhibits EGFand PDGF-induced MAPK signaling in glioblastoma cells, whereas the UV-induced JNK signaling is not suppressed (93). Further, the transcription factor c-Jun/AP-1 can in turn regulate the transcription of PTEN. The $c$ - $J u n^{-/-}$fibroblasts display elevated mRNA and protein levels of PTEN and concomitant inactivation of Akt with prolonged survival in cancer cells with either null or wild-type p53, suggesting that PTEN is negatively regulated by c-Jun in a p53-independent manner (94). Moreover, it has been reported that c-Jun inhibits PTEN transcription through binding to the PF-1 site in the promoter sequence of PTEN (94).

\section{Conclusion}

The molecular mechanism of PI3K/Akt and JNK pathway activation is now well understood. Genetic alternations frequently occur within PI3K/Akt signaling pathway in cancers, leading to the constitutively active PI3K/Akt signaling, and then promoting cell proliferation, survival, migration and invasion through interacting with downstream effectors. JNK signaling pathway is one of the downstream pathways of PI3K/Akt signaling, and plays dual roles in apoptosis in a stimulus-dependent manner. The specific role that JNK takes on, in apoptosis determines its involvement in tumor development, as well as different interplays between these two pathways. Activation of PI3K/Akt signaling can inhibit stress- and cytokine-induced JNK activation through
Akt antagonizing the formation of the JIP1-JNK module, as well as the activities of upstream kinases ASK1, MKK4/7 and MLK. On the other hand, activation of JNK pathway is concomitant with activation of PI3K/Akt pathway in cancer in the context of EGFR overexpression stimulation or PTEN deficiency. Hence, the interaction between PI3K/Akt and JNK pathways provides potential targets and therapeutic strategies for cancer treatment. Inhibitors aimed at PI3K/Akt and JNK pathways simultaneously may have synergistic positive effects on improving the survival and prognosis of cancer patients, which should be further investigated.

\section{Acknowledgements}

This study was supported by a Central Research Grant from the Hong Kong Polytechnic University (RTH4).

\section{References}

1. Cantley LC: The phosphoinositide 3-kinase pathway. Science 296: 1655-1657, 2002.

2. Katso R, Okkenhaug K, Ahmadi K, White S, Timms J and Waterfield MD: Cellular function of phosphoinositide 3-kinases: Implications for development, homeostasis, and cancer. Annu Rev Cell Dev Biol 17: 615-675, 2001.

3. Vanhaesebroeck B and Alessi DR: The PI3K-PDK1 connection: More than just a road to PKB. Biochem J 346: 561-576, 2000.

4. Toker A and Newton AC: Cellular signaling: Pivoting around PDK-1. Cell 103: 185-188, 2000.

5. Sarbassov DD, Ali SM and Sabatini DM: Growing roles for the mTOR pathway. Curr Opin Cell Biol 17: 596-603, 2005.

6. Vivanco I and Sawyers CL: The phosphatidylinositol 3-kinase AKT pathway in human cancer. Nat Rev Cancer 2: 489-501, 2002.

7. Sun H, Lesche R, Li DM, Liliental J, Zhang H, Gao J, Gavrilova N, Mueller B, Liu X and Wu H: PTEN modulates cell cycle progression and cell survival by regulating phosphatidylinositol 3,4,5,-trisphosphate and Akt/protein kinase B signaling pathway. Proc Natl Acad Sci USA 96: 6199-6204, 1999.

8. Song MS, Salmena L and Pandolfi PP: The functions and regulation of the PTEN tumour suppressor. Nat Rev Mol Cell Biol 13: 283-296, 2012.

9. Srividya MR, Thota B, Shailaja BC, Arivazhagan A, Thennarasu K, Chandramouli BA, Hegde AS and Santosh V: Homozygous 10q23/ PTEN deletion and its impact on outcome in glioblastoma: A prospective translational study on a uniformly treated cohort of adult patients. Neuropathology 31: 376-383, 2011.

10. Chong ML, Loh M, Thakkar B, Pang B, Iacopetta B and Soong R: Phosphatidylinositol-3-kinase pathway aberrations in gastric and colorectal cancer: Meta-analysis, co-occurrence and ethnic variation. Int J Cancer 134: 1232-1238, 2014.

11. Garcia-Dios DA, Lambrechts D, Coenegrachts L, Vandenput I, Capoen A, Webb PM, Ferguson K, Akslen LA, Claes B, Vergote I, et al; ANECS: High-throughput interrogation of PIK3CA, PTEN, KRAS, FBXW7 and TP53 mutations in primary endometrial carcinoma. Gynecol Oncol 128: 327-334, 2013.

12. McConechy MK, Ding J, Senz J, Yang W, Melnyk N, Tone AA, Prentice LM, Wiegand KC, McAlpine JN, Shah SP, et al: Ovarian and endometrial endometrioid carcinomas have distinct CTNNB1 and PTEN mutation profiles. Mod Pathol 27: 128-134, 2014.

13. Jin G, Kim MJ, Jeon HS, Choi JE, Kim DS, Lee EB, Cha SI, Yoon GS, Kim CH and Jung TH: PTEN mutations and relationship to EGFR, ERBB2, KRAS, and TP53 mutations in non-small cell lung cancers. Lung Cancer 69: 279-283, 2010.

14. Kang-Park S, Lee YI and Lee YI: PTEN modulates insulin-like growth factor II (IGF-II)-mediated signaling; the protein phosphatase activity of PTEN downregulates IGF-II expression in hepatoma cells. FEBS Lett 545: 203-208, 2003.

15. Yi HK, Kim SY, Hwang PH, Kim CY, Yang DH, Oh Y and Lee DY: Impact of PTEN on the expression of insulin-like growth factors (IGFs) and IGF-binding proteins in human gastric adenocarcinoma cells. Biochem Biophys Res Commun 330: 760-767, 2005. 
16. Oki E, Baba H, Tokunaga E, Nakamura T, Ueda N, Futatsugi M, Mashino K, Yamamoto M, Ikebe M, Kakeji Y, et al: Akt phosphorylation associates with LOH of PTEN and leads to chemoresistance for gastric cancer. Int J Cancer 117: 376-380, 2005.

17. Tang JM, He QY, Guo RX and Chang XJ: Phosphorylated Akt overexpression and loss of PTEN expression in non-small cell lung cancer confers poor prognosis. Lung Cancer 51: 181-191, 2006.

18. Vivanco I, Rohle D, Versele M, Iwanami A, Kuga D, Oldrini B, Tanaka K, Dang J, Kubek S, Palaskas N, et al: The phosphatase and tensin homolog regulates epidermal growth factor receptor (EGFR) inhibitor response by targeting EGFR for degradation. Proc Natl Acad Sci USA 107: 6459-6464, 2010.

19. Weston CR and Davis RJ: The JNK signal transduction pathway. Curr Opin Cell Biol 19: 142-149, 2007.

20. Bode AM and Dong Z: The functional contrariety of JNK. Mol Carcinog 46: 591-598, 2007.

21. Yang JY, Moulin N, van Bemmelen MX, Dubuis G, Tawadros T, Haefliger JA, Waeber G and Widmann C: Splice variant-specific stabilization of JNKs by IB1/JIP1. Cell Signal 19: 2201-2207, 2007.

22. Davis RJ: Signal transduction by the JNK group of MAP kinases. Cell 103: 239-252, 2000.

23. Barr RK and Bogoyevitch MA: The c-Jun N-terminal protein kinase family of mitogen-activated protein kinases (JNK MAPKs). Int J Biochem Cell Biol 33: 1047-1063, 2001.

24. Tournier C, Dong C, Turner TK, Jones SN, Flavell RA and Davis RJ: MKK7 is an essential component of the JNK signal transduction pathway activated by proinflammatory cytokines. Genes Dev 15: 1419-1426, 2001.

25. Haeusgen W, Herdegen T and Waetzig V: The bottleneck of JNK signaling: Molecular and functional characteristics of MKK4 and MKK7. Eur J Cell Biol 90: 536-544, 2011

26. Leicht DT, Balan V, Kaplun A, Singh-Gupta V, Kaplun L, Dobson $\mathrm{M}$ and Tzivion G: Raf kinases: Function, regulation and role in human cancer. Biochim Biophys Acta 1773: 1196-1212, 2007.

27. Ichijo H, Nishida E, Irie K, ten Dijke P, Saitoh M, Moriguchi T, Takagi M, Matsumoto K, Miyazono K and Gotoh Y: Induction of apoptosis by ASK1, a mammalian MAPKKK that activates SAPK/JNK and p38 signaling pathways. Science 275: 90-94, 1997.

28. Sun BK, Kim JH, Nguyen HN, Oh S, Kim SY, Choi S, Choi HJ, Lee YJ and Song JJ: MEKK1/MEKK4 are responsible for TRAIL-induced JNK/p38 phosphorylation. Oncol Rep 25: 537-544, 2011

29. Xu Z, Maroney AC, Dobrzanski P, Kukekov NV and Greene LA: The MLK family mediates c-Jun N-terminal kinase activation in neuronal apoptosis. Mol Cell Biol 21: 4713-4724, 2001.

30. Lopez-Ilasaca M: Signaling from G-protein-coupled receptors to mitogen-activated protein (MAP)-kinase cascades. Biochem Pharmacol 56: 269-277, 1998.

31. Shaulian E: AP-1 - The Jun proteins: Oncogenes or tumor suppressors in disguise? Cell Signal 22: 894-899, 2010.

32. Whitmarsh AJ, Cavanagh J, Tournier C, Yasuda J and Davis RJ: A mammalian scaffold complex that selectively mediates MAP kinase activation. Science 281: 1671-1674, 1998.

33. Whitmarsh AJ, Kuan CY, Kennedy NJ, Kelkar N, Haydar TF, Mordes JP, Appel M, Rossini AA, Jones SN, Flavell RA, et al: Requirement of the JIP1 scaffold protein for stress-induced JNK activation. Genes Dev 15: 2421-2432, 2001.

34. Nihalani D, Meyer D, Pajni S and Holzman LB: Mixed lineage kinase-dependent JNK activation is governed by interactions of scaffold protein JIP with MAPK module components. EMBO J 20: 3447-3458, 2001.

35. Lin A: Activation of the JNK signaling pathway: Breaking the brake on apoptosis. BioEssays 25: 17-24, 2003

36. Lin A and Dibling B: The true face of JNK activation in apoptosis. Aging Cell 1: 112-116, 2002.

37. Besirli CG and Johnson EM Jr: JNK-independent activation of c-Jun during neuronal apoptosis induced by multiple DNA-damaging agents. J Biol Chem 278: 22357-22366, 2003.

38. Huntwork-Rodriguez S, Wang B, Watkins T, Ghosh AS Pozniak CD, Bustos D, Newton K, Kirkpatrick DS and Lewcock JW: JNK-mediated phosphorylation of DLK suppresses its ubiquitination to promote neuronal apoptosis. J Cell Biol 202: 747-763, 2013.

39. Reno EM, Haughian JM, Jackson TA, Thorne AM and Bradford AP: c-Jun N-terminal kinase regulates apoptosis in endometrial cancer cells. Apoptosis 14: 809-820, 2009.
40. Robitaille K, Daviau A, Lachance G, Couture JP and Blouin R: Calphostin C-induced apoptosis is mediated by a tissue transglutaminase-dependent mechanism involving the DLK/JNK signaling pathway. Cell Death Differ 15: 1522-1531, 2008.

41. Song J, Ko HS, Sohn EJ, Kim B, Kim JH, Kim HJ, Kim C, $\mathrm{Kim} \mathrm{JE}$ and Kim SH: Inhibition of protein kinase $\mathrm{C} \alpha / \beta \mathrm{II}$ and activation of c-Jun NH2-terminal kinase mediate glycyrrhetinic acid induced apoptosis in non-small cell lung cancer NCI-H460 cells. Bioorg Med Chem Lett 24: 1188-1191, 2014.

42. Kim BJ, Ryu SW and Song BJ: JNK- and p38 kinase-mediated phosphorylation of Bax leads to its activation and mitochondrial translocation and to apoptosis of human hepatoma HepG2 cells J Biol Chem 281: 21256-21265, 2006.

43. Ambacher KK, Pitzul KB, Karajgikar M, Hamilton A, Ferguson SS and Cregan SP: The JNK- and AKT/GSK3 $\beta$ signaling pathways converge to regulate Puma induction and neuronal apoptosis induced by trophic factor deprivation. PLoS One 7: e46885, 2012.

44. Zhao Z, Wang J, Tang J, Liu X, Zhong Q, Wang F, Hu W, Yuan Z, Nie $C$ and Wei Y: JNK- and Akt-mediated Puma expression in the apoptosis of cisplatin-resistant ovarian cancer cells. Biochem J 444: 291-301, 2012

45. Yu J and Zhang L: PUMA, a potent killer with or without p53. Oncogene 27 (Suppl 1): S71-S83, 2008.

46. Buschmann T, Potapova O, Bar-Shira A, Ivanov VN, Fuchs SY, Henderson S, Fried VA, Minamoto T, Alarcon-Vargas D, Pincus MR, et al: Jun NH2-terminal kinase phosphorylation of p53 on Thr-81 is important for p53 stabilization and transcriptional activities in response to stress. Mol Cell Biol 21: 2743-2754, 2001.

47. Jones EV, Dickman MJ and Whitmarsh AJ: Regulation of p73-mediated apoptosis by c-Jun N-terminal kinase. Biochem J 405: 617-623, 2007.

48. Yu C, Minemoto Y, Zhang J, Liu J, Tang F, Bui TN, Xiang J and Lin A: JNK suppresses apoptosis via phosphorylation of the proapoptotic Bcl-2 family protein BAD. Mol Cell 13: 329-340, 2004.

49. Nishina H, Fischer KD, Radvanyi L, Shahinian A, Hakem R, Rubie EA, Bernstein A, Mak TW, Woodgett JR and Penninger JM: Stress-signalling kinase Sek1 protects thymocytes from apoptosis mediated by CD95 and CD3. Nature 385: 350-353, 1997.

50. Potapova O, Gorospe M, Dougherty RH, Dean NM, Gaarde WA and Holbrook NJ: Inhibition of c-Jun N-terminal kinase 2 expression suppresses growth and induces apoptosis of human tumor cells in a p53-dependent manner. Mol Cell Biol 20: 1713-1722, 2000.

51. Cellurale C, Sabio G, Kennedy NJ, Das M, Barlow M, Sandy P, Jacks T and Davis RJ: Requirement of c-Jun NH(2)-terminal kinase for Ras-initiated tumor formation. Mol Cell Biol 31: 1565-1576, 2011.

52. Xiao L and Lang W: A dominant role for the c-Jun NH2-terminal kinase in oncogenic ras-induced morphologic transformation of human lung carcinoma cells. Cancer Res 60: 400-408, 2000

53. Nielsen C, Thastrup J, Bøttzauw T, Jäättelä M and Kallunki T: c-Jun NH2-terminal kinase 2 is required for Ras transformation independently of activator protein 1. Cancer Res 67: 178-185, 2007.

54. Mathiasen DP, Egebjerg C, Andersen SH, Rafn B, Puustinen P, Khanna A, Daugaard M, Valo E, Tuomela S, Bøttzauw T, et al: Identification of a c-Jun N-terminal kinase-2-dependent signal amplification cascade that regulates c-Myc levels in ras transformation. Oncogene 31: 390-401, 2012.

55. Johnson R, Spiegelman B, Hanahan D and Wisdom R: Cellular transformation and malignancy induced by ras require c-jun. Mol Cell Biol 16: 4504-4511, 1996.

56. Shibata W, Maeda S, Hikiba Y, Yanai A, Sakamoto K, Nakagawa H, Ogura K, Karin M and Omata M: c-Jun $\mathrm{NH} 2$-terminal kinase 1 is a critical regulator for the development of gastric cancer in mice. Cancer Res 68: 5031-5039, 2008.

57. Chang Q, Chen J, Beezhold KJ, Castranova V, Shi X and Chen F: JNK1 activation predicts the prognostic outcome of the human hepatocellular carcinoma. Mol Cancer 8: 64, 2009.

58. Wang X, Chao L, Li X, Ma G, Chen L, Zang Y and Zhou G: Elevated expression of phosphorylated c-Jun NH2-terminal kinase in basal-like and 'triple-negative' breast cancers. Hum Pathol 41: 401-406, 2010

59. Li JY, Wang H, May S, Song X, Fueyo J, Fuller GN and Wang H: Constitutive activation of c-Jun N-terminal kinase correlates with histologic grade and EGFR expression in diffuse gliomas. J Neurooncol 88: 11-17, 2008. 
60. Takahashi R, Hirata Y, Sakitani K, Nakata W, Kinoshita H, Hayakawa Y, Nakagawa H, Sakamoto K, Hikiba Y, Ijichi H, et al: Therapeutic effect of c-Jun N-terminal kinase inhibition on pancreatic cancer. Cancer Sci 104: 337-344, 2013.

61. Chen P,O'Neal JF,Ebelt ND, Cantrell MA,MitraS, Nasrazadani A, Vandenbroek TL, Heasley LE and Van Den Berg CL: Jnk2 effects on tumor development, genetic instability and replicative stress in an oncogene-driven mouse mammary tumor model. PLoS One 5: e10443, 2010.

62. Das M, Garlick DS, Greiner DL and Davis RJ: The role of JNK in the development of hepatocellular carcinoma. Genes Dev 25 634-645, 2011

63. Kennedy NJ, Sluss HK, Jones SN, Bar-Sagi D, Flavell RA and Davis RJ: Suppression of Ras-stimulated transformation by the JNK signal transduction pathway. Genes Dev 17: 629-637, 2003

64. Hübner A, Mulholland DJ, Standen CL, Karasarides M Cavanagh-Kyros J, Barrett T, Chi H, Greiner DL, Tournier C, Sawyers CL, et al: JNK and PTEN cooperatively control the development of invasive adenocarcinoma of the prostate. Proc Natl Acad Sci USA 109: 12046-12051, 2012.

65. Ying J, Li H, Cui Y, Wong AH, Langford C and Tao Q Epigenetic disruption of two proapoptotic genes MAPK10/JNK3 and PTPN13/FAP-1 in multiple lymphomas and carcinomas through hypermethylation of a common bidirectional promoter. Leukemia 20: 1173-1175, 2006

66. Yoshida S, Fukino K, Harada H, Nagai H, Imoto I, Inazawa J, Takahashi H, Teramoto A and Emi M: The c-Jun NH2-terminal kinase3 (JNK3) gene: Genomic structure, chromosomal assignment, and loss of expression in brain tumors. J Hum Genet 46: $182-187,2001$

67. Jurewicz A, Matysiak M, Tybor K and Selmaj K: TNF-induced death of adult human oligodendrocytes is mediated by c-jun NH2-terminal kinase-3. Brain 126: 1358-1370, 2003.

68. Dajas-Bailador F, Bantounas I, Jones EV and Whitmarsh AJ: Regulation of axon growth by the JIP1-AKT axis. J Cell Sci 127: 230-239, 2014

69. Kim AH, Sasaki T and Chao MV: JNK-interacting protein 1 promotes Akt1 activation. J Biol Chem 278: 29830-29836, 2003.

70. Pan J, Pei DS, Yin XH, Hui L and Zhang GY: Involvement of oxidative stress in the rapid Aktl regulating a JNK scaffold during ischemia in rat hippocampus. Neurosci Lett 392: 47-51, 2006.

71. Kim AH, Yano H, Cho H, Meyer D, Monks B, Margolis B, Birnbaum MJ and Chao MV: Akt1 regulates a JNK scaffold during excitotoxic apoptosis. Neuron 35: 697-709, 2002

72. Cerezo A, Martínez-A C, Lanzarot D, Fischer S, Franke TF and Rebollo A: Role of Akt and c-Jun N-terminal kinase 2 in apoptosis induced by interleukin-4 deprivation. Mol Biol Cell 9: 3107-3118, 1998

73. Levresse V, Butterfield L, Zentrich E and Heasley LE: Akt negatively regulates the cJun N-terminal kinase pathway in PC12 cells. J Neurosci Res 62: 799-808, 2000.

74. Okubo Y, Blakesley VA, Stannard B, Gutkind S and Le Roith D Insulin-like growth factor-I inhibits the stress-activated protein kinase/c-Jun N-terminal kinase. J Biol Chem 273: 25961-25966, 1998.

75. Wang Q, Zhang QG, Wu DN, Yin XH and Zhang GY: Neuroprotection of selenite against ischemic brain injury through negatively regulating early activation of ASK1/JNK cascade via activation of PI3K/AKT pathway. Acta Pharmacol Sin 28: 19-27, 2007.

76. Kim AH, Khursigara G, Sun X, Franke TF and Chao MV: Akt phosphorylates and negatively regulates apoptosis signalregulating kinase 1. Mol Cell Biol 21: 893-901, 2001.

77. Aikin R, Maysinger D and Rosenberg L: Cross-talk between phosphatidylinositol 3-kinase/AKT and c-jun NH2-terminal kinase mediates survival of isolated human islets. Endocrinology 145: 4522-4531, 2004

78. Xie D, Gore C, Zhou J, Pong RC, Zhang H, Yu L, Vessella RL, Min W and Hsieh JT: DAB2IP coordinates both PI3K-Akt and ASK1 pathways for cell survival and apoptosis. Proc Natl Acad Sci USA 106: 19878-19883, 2009.
79. Park HS, Kim MS, Huh SH, Park J, Chung J, Kang SS and Choi EJ: Akt (protein kinase B) negatively regulates SEK1 by means of protein phosphorylation. J Biol Chem 277: 2573-2578, 2002.

80. Murakami T, Takagi H, Suzuma K, Suzuma I, Ohashi H, Watanabe D, Ojima T, Suganami E, Kurimoto M, Kaneto H, et al: Angiopoietin-1 attenuates $\mathrm{H}_{2} \mathrm{O}_{2}$-induced SEK1/JNK phosphorylation through the phosphatidylinositol 3-kinase/Akt pathway in vascular endothelial cells. J Biol Chem 280: 31841-31849, 2005.

81. Barthwal MK, Sathyanarayana P, Kundu CN, Rana B, Pradeep A, Sharma C, Woodgett JR and Rana A: Negative regulation of mixed lineage kinase 3 by protein kinase B/AKT leads to cell survival. J Biol Chem 278: 3897-3902, 2003.

82. Wen XR, Li C, Zong YY, Yu CZ, Xu J, Han D and Zhang GY: Dual inhibitory roles of geldanamycin on the c-Jun NH2-terminal kinase 3 signal pathway through suppressing the expression of mixed-lineage kinase 3 and attenuating the activation of apoptosis signal-regulating kinase 1 via facilitating the activation of Akt in ischemic brain injury. Neuroscience 156: 483-497, 2008.

83. Song JJ and Lee YJ: Dissociation of Akt1 from its negative regulator JIP1 is mediated through the ASK1-MEK-JNK signal transduction pathway during metabolic oxidative stress: A negative feedback loop. J Cell Biol 170: 61-72, 2005.

84. Logan SK, Falasca M, Hu P and Schlessinger J: Phosphatidylinositol 3-kinase mediates epidermal growth factor-induced activation of the c-Jun N-terminal kinase signaling pathway. Mol Cell Biol 17: 5784-5790, 1997.

85. Vivanco I, Palaskas N, Tran C, Finn SP, Getz G, Kennedy NJ, Jiao J, Rose J, Xie W, Loda M, et al: Identification of the JNK signaling pathway as a functional target of the tumor suppressor PTEN. Cancer Cell 11: 555-569, 2007.

86. Cui J, Han SY, Wang C, Su W, Harshyne L, Holgado-Madruga M and Wong AJ: c-Jun NH(2)-terminal kinase 2 alpha2 promotes the tumorigenicity of human glioblastoma cells. Cancer Res 66: 10024-10031, 2006.

87. Tsuiki H, Tnani M, Okamoto I, Kenyon LC, Emlet DR, HolgadoMadruga M, Lanham IS, Joynes CJ, Vo KT and Wong AJ: Constitutively active forms of c-Jun NH2-terminal kinase are expressed in primary glial tumors. Cancer Res 63: 250-255, 2003.

88. Nitta RT, Del Vecchio CA, Chu AH, Mitra SS, Godwin AK and Wong AJ: The role of the c-Jun N-terminal kinase 2 - $\alpha$-isoform in non-small cell lung carcinoma tumorigenesis. Oncogene 30: 234-244, 2011.

89. Antonyak MA, Kenyon LC, Godwin AK, James DC, Emlet DR, Okamoto I, Tnani M, Holgado-Madruga M, Moscatello DK and Wong AJ: Elevated JNK activation contributes to the pathogenesis of human brain tumors. Oncogene 21: 5038-5046, 2002.

90. Bost F, McKay R, Bost M, Potapova O, Dean NM and Mercola D: The Jun kinase 2 isoform is preferentially required for epidermal growth factor-induced transformation of human A549 lung carcinoma cells. Mol Cell Biol 19: 1938-1949, 1999.

91. Rong Y, Belozerov VE, Tucker-Burden C, Chen G, Durden DL, Olson JJ, Van Meir EG, Mackman N and Brat DJ: Epidermal growth factor receptor and PTEN modulate tissue factor expression in glioblastoma through JunD/activator protein-1 transcriptional activity. Cancer Res 69: 2540-2549, 2009.

92. Bonavia R, Inda MM, Vandenberg S, Cheng SY, Nagane M, Hadwiger P, Tan P, Sah DW, Cavenee WK and Furnari FB: EGFRvIII promotes glioma angiogenesis and growth through the NF- $\mathrm{kB}$, interleukin-8 pathway. Oncogene 31: 4054-4066, 2012.

93. Gu J, Tamura M and Yamada KM: Tumor suppressor PTEN inhibits integrin- and growth factor-mediated mitogen-activated protein (MAP) kinase signaling pathways. J Cell Biol 143: 1375-1383, 1998.

94. Hettinger K, Vikhanskaya F, Poh MK, Lee MK, de Belle I, Zhang JT, Reddy SA and Sabapathy K: c-Jun promotes cellular survival by suppression of PTEN. Cell Death Differ 14: 218-229, 2007. 